\title{
O PAPEL DE SOCIÓLOGOS EM PROJETOS DE INTERVENÇÃO SOCIAL \\ A experiência de docentes e investigadores da Universidade de Évora ao longo de vinte anos
}

\section{THE ROLE OF SOCIOLOGISTS IN SOCIAL INTERVENTION PROJECTS}

\section{The experience of teachers and researchers at the University of Évora over twenty years}

\author{
Marcos Santos \\ Universidade de Évora, Centro Interdisciplinar de Ciências Sociais - CICS.NOVA.UÉvora, Universidade de \\ Évora, Palácio do Vimioso, Gab. 108, Largo Marquês de Marialva, 8, 7000-809 Évora, Portugal. \\ Email:mosantos@uevora.pt

\section{Maria da Saudade Baltazar} \\ Universidade de Évora, Escola de Ciências Sociais, Departamento de Sociologia \& Universidade de Évora, Centro \\ Interdisciplinar de Ciências Sociais - CICS.NOVA.UÉvora. Universidade de Évora, Palácio do Vimioso, Gab. \\ 108, Largo Marquês de Marialva, 8, 7000-809 Évora, Portugal. Email: baltazar@uevora.pt
}

\begin{abstract}
Resumo: O objetivo do trabalho que se apresenta consiste em divulgar conhecimentos, experiências e reflexões que os autores sedimentaram ao longo da sua participação em projetos, financiados pela União Europeia e por Programas nacionais. Também se visa colocar em debate várias considerações sobre a adequação da formação de base dos diplomados em Sociologia, sobre lacunas na sua formação em hard skills, e sobre a importância de se cultivar alguma(s) das soft skills exigidas aos futuros profissionais para o desempenho de funções em projetos de intervenção social.
\end{abstract}

Palavras-chave: projetos de intervenção local, sociólogos, competências técnicas, competências não técnicas.

\begin{abstract}
The objective of this work is to disseminate knowledge, experiences and reflections that the authors consolidated throughout their participation in projects financed by the European Union and by national programs. It also aims to debate several considerations on the adequacy of the basic training of graduates in Sociology, gaps in our training in hard skills, and the importance of acquiring soft skills required for future professionals in social intervention projects.
\end{abstract}

Keywords: local intervention projects, sociologists, technical skills, non-technical skills.

\section{Introdução}

Na sequência do apelo ao envio de propostas de comunicações/posters por parte da Associação Portuguesa de Sociologia sobre o trabalho dos sociólogos envolvidos em funções e programas de intervenção local, os autores apresentam neste texto os seus contributos recolhidos sobre essa temática ao longo de 
cerca vinte anos na qualidade de participantes/intervenientes, docentes e investigadores.

O objetivo do trabalho que se apresenta consiste, assim em divulgar conhecimentos, experiências e reflexões que os autores retiveram da sua participação em projetos Equal, e também da participação em outros projetos, implementados nomeadamente ao abrigo dos seguintes Programas: Luta Contra a Pobreza, Inclusão e Desenvolvimento, Ser Criança e Interreg.

A título de exemplo, são apresentados o projeto concretizado ao abrigo do Programa Inter-regional de Cooperação INTERREG IVC (projeto Winnet 8 - Promoção da Inovação em Igualdade de Género na Europa), e o projeto Qual_IS Qualificar a Intervenção Social, financiado pelo POPH - Programa de Formação-Ação para Entidades da Economia Social Regiões de Portugal: Norte/Centro/Alentejo e promovido pela EAPN (Rede Europeia Anti-Pobreza/Portugal).

Complementarmente, colocam-se em debate várias considerações sobre a adequação da formação de base dos diplomados em Sociologia, sobre lacunas na formação em hard skills, e sobre a importância de se cultivar (quer em várias unidades curriculares do curso, quer em atividades extra curriculares), alguma(s) das soft skills exigidas para o desempenho de funções em projetos de intervenção social.

\section{Métodos}

Os procedimentos metodológicos adotados consistiram na sistematização da observação participante, que teve lugar ao longo dos ciclos de preparação e vigência de projetos de intervenção social em que os autores participaram, e na revisitação crítica de textos (diagnósticos, relatórios de execução, estudos, relatórios de avaliação, etc.), redigidos ou coordenados principalmente pelos autores, ou por outros membros que integraram as equipas dos referidos projetos.

\section{Sociologia de ação: breve enquadramento da intervenção sociológica}

Os primórdios da sociologia da intervenção situam-se no século XIX, associada a uma sociologia de campo, com análises descritivas de usos, costumes, tradições, funcionamento de instituições e de grupos, e formulação de tipologias a partir de observações detalhadas para apresentar e compreender os fenómenos sociais. Os trabalhos monográficos de microssociologia de Frederic Le Play (1862) assumidos pela sociologia empírica norte-americana, nomeadamente pela Escola de Chicago, 
foram reforçados no espaço europeu com as análises institucionais e estratégicas assim como pelo denominado intervencionismo sociológico (Guerra, 2003; Herreros, 2013; Moreau, 2015).

Se num primeiro momento, a dificuldade de conjugar teoria e ação, indivíduo e sociedade esteve na base da intervenção sociológica, na década de 70 o foco foi encaminhado para uma sociologia mais crítica, que se recentra numa abordagem racional dos atores para definir estratégias adequadas às metas a alcançar tendo em conta os objetivos traçados, minimizando/anulando os pontos fracos e potenciando os pontos fortes, numa relação direta entre sociólogo e população em estudo.

Já as dinâmicas socioeconómicas ocorridas nas últimas décadas resultantes da intensificação do processo de globalização, da expansão económica, da crise de legitimidade dos Estados soberanos, de crises económico-sociais associadas ao acentuar de problemas socais como desemprego, pobreza, exclusão social e migrações, tendem a potenciar este tipo de intervenções sociais e que visam a mudança social.

Para Isabel Guerra, "a sociologia de intervenção sempre colocou os seus desafios na procura utópica da chave da mudança social e, através dessa reflexão no ensaio de métodos de redução de incertezas" (Guerra, 2003, p. 73). A mesma autora acrescenta que a profissionalização crescente das ciências sociais, com particular destaque na área da sociologia e da antropologia, tem suscitado o interesse por uma "teoria da ação" em busca de uma outra relação entre intervenção e conhecimento que minimize a fragmentação crescente e diversidade dos domínios de atividade profissional.

Ou seja, a sociologia da ação inscreve-se numa lógica própria de um espaço de interseção, que resulta do facto de congregar a produção e a utilização imediata, e que não corresponde nem a uma metodologia da investigação "pura" nem mesmo às simples regras de ação imediata, numa constante busca de equilíbrio e sem tentações hegemónicas de qualquer uma das duas vertentes. De algum modo essa sociologia surge associada à necessária desocultação e transparência dos agentes e processos envolvido na mudança social.

Assim, a sociologia da intervenção, segundo Vranken \& Olgierd (2001), corresponde a um exercício profissional (epistemologia, metodologia e papel do sociólogo-interventor) que determina o posicionamento da componente prática, a partir da solicitação do sistema-cliente, numa situação de interação entre atores, visando o cumprimento de uma missão.

Em Portugal, em especial a partir de 1974, o reforço da formação em sociologia e correspondente aumento de sociólogos, conduziram à diversificação das suas áreas de atuação, com participação muitas vezes em trabalhos de equipa com 
profissionais de outras áreas de formação, e com um interesse crescente no desenvolvimento de estudos prévios à fase da intervenção.

Mas este recrudescimento da investigação-ação, que tem vindo a ocorrer a nível nacional e internacional, encontra-se, no entanto, associado a críticas pouco consistentes, formuladas em alguns circuitos científicos. Ou seja, se Kurt Lewin, em 1948, lançou a action reserch, desde então não tem sido suficientemente praticada e divulgada para além da esfera de atuação dos que utilizam os seus resultados, não obstante as ciências sociais terem procurado retirar benefícios do contexto vigente de I\&D (investigação e desenvolvimento), o qual tem beneficiado de concessão de financiamento destinado a este tipo de intervenções (Almeida, 2001).

O mesmo autor defende ainda que o carácter pluriparadigmático das ciências sociais associado à fraca divulgação da prática da investigação-ação condiciona a existência de um verdadeiro paradigma neste domínio. O nexo entre conhecimento e ação revela-se pouco linear e direto, por corresponder a uma lógica própria de um projeto de investigação-ação numa relação cognitiva de apoio ao seu promotor/financiador, na qual o sociólogo assume desenvolver um produto de utilidade prática.

Em suma, e de uma forma sucinta, pode-se afirmar que se está perante uma metodologia de investigação orientada para a melhoria da prática nos vários domínios de ação. O duplo objetivo que aqui se reconhece, é por um lado facilitar o aperfeiçoamento das pessoas e dos grupos com quem se trabalha e, por outro potenciar os resultados obtidos naquilo que se faz. Esta metodologia visa a melhoria das práticas através da mudança e da aprendizagem a partir dos efeitos de tais mudanças, assim como potencia a participação de todos os implicados.

De acordo com Trilla (1998), desenvolve-se numa espiral de ciclos de planificação, ação, observação e reflexão, correspondendo a um processo sistemático de aprendizagem orientado para a praxis, e que pressupõe que esta seja submetida à prova, apresentando uma justificação a partir do trabalho executado, mediante uma argumentação desenvolvida, comprovada e cientificamente examinada.

Assim, tendo por base a ideia de que o conhecimento (no seu amplo sentido, tácito e explícito) é socialmente condicionado, a sociologia do conhecimento (Bourdieu, 2001; Mannhein, 1974), apesar de lhe serem reconhecidas algumas contradições teóricas e metodológicas, identifica referenciais para a reflexão sobre a profissão do sociólogo, nomeadamente no que se prende com a ação.

Verifica-se, portanto, que existe uma relação dialética entre estes dois domínios, o que está patente neste texto onde se mostra que tendo nós partido do conhecimento disponível para aplicar à prática, a experiência no terreno permitiu confirmar e reajustar as bases do nosso ponto de partida. 
Perante a existência de várias correntes da sociologia da ação (objectivistas dos anos 60, subjectivista dos anos 70, construtivista dos anos 80, e ecológica dos anos 90) referidas por Guerra (2003), no caso presente foi privilegiado o modelo ecológico (corrente ecológica), simultaneamente multireferencial e sistémico que situa a intervenção social, nos sistemas família, indivíduo e comunidade.

O modelo referido requer a aplicação de uma metodologia participativa de projeto visando o empenho esclarecido dos participantes na intervenção, o que pode ser levado a cabo através de um conjunto alargado de ferramentas, de entre as quais foram aplicadas nos projetos em análise as seguintes: Brainstorming, Comunidades de prática, World Café e Focus Group.

Estas orientações metodológicas são sistematizadas de seguida, a partir de experiências e práticas vivenciadas no acompanhamento de projetos de intervenção social.

\section{Considerações e aprendizagens em torno da intervenção}

Elegemos como temática a experiência na intervenção social através de projectos, pelo que apresentamos um modelo sucinto que possibilite aprofundar o raciocínio que nos propomos seguir.

Tabela 1 O processo de intervenção (resumido/simplificado/abreviado)

A decisão de intervir, a preparação e o desenho da intervenção, com base em problemas/necessidades por resolver

(incluindo preenchimento e submissão da candidatura a uma fonte de financiamento)

A concretização, monitorização e avaliação

Fonte: elaboração própria.

O delineamento deste esquema decorreu da aprendizagem resultante da análise de modelos subjacentes a processos de intervenção, com os quais os autores contactaram ao longo da docência e da participação em projectos, modelos que têm surgido, ou sido aplicados, em vários pontos do globo, devido a especificidades várias tais como: contextos ou zonas de intervenção peculiares, experiências diversificadas, problemáticas diferenciadas, etc. Entre esses modelos de intervenção, 1 que compartilham no entanto de algumas características comuns, podem ser recenseados, de acordo com Santos (2011), os seguintes: i) Gestão de Ciclo do Projeto 
(GCP); ii) Método do Quadro Lógico (MQL); iii) Método Aplicado de Planeamento e Avaliação de Projetos (MAPA); iv) Metodologia Ziel Orientierte Projekt Planung (ZOPP); v) Metodologia de Planeamento de Projetos por Objetivos (MPPO); vi) Método Altadir de Planeamento Popular (MAPP); Metodologia da Faculdade Latino Americana de Ciências Sociais (FLACSO); vii) Modelo PRECEDE-PROCEED (um dos principais modelos de intervenção de promoção da saúde); viii) Planeamento Estratégico Situacional (PES); ix) Planeamento Estratégico-Participativo, e x) Modelo Interativo de Planificação de Programas (MIPP).

O modelo apresentado acima acolhe uma chamada de atenção para a fase anterior à intervenção, na qual os responsáveis por uma organização tomam a decisão de intervir, lançando o repto a outras organizações. Esta é uma fase importante que influencia o futuro da intervenção devido às opções que são tomadas, incidindo entre outras sobre as seguintes questões: Porque se intervém? Para quem? Com quem? Como?

Por terem sido confrontados com insuficiências de informação e de conhecimentos pertinentes que enfermaram alguns dos projetos nos quais participaram, em especial nos momentos de avaliação, os autores têm recorrido nomeadamente na fase inicial, à utilização da checklist 9W2H (adaptada da checklist 5W2H), ${ }^{2}$ aquela também designada pelo método dos 12 ajudantes e explanada na tabela seguinte.

Tabela 2 Método dos 12 ajudantes

\begin{tabular}{|c|c|c|c|}
\hline \multicolumn{4}{|c|}{ Problematização das Questões de Partida } \\
\hline № & Designação & Formulação & Checklist (10W2H) \\
\hline 1 & Natureza da iniciativa & O que se quer fazer? & What we want to do? \\
\hline 2 & Origem e fundamentação & Porque se quer fazer? & Why do we want to do it? \\
\hline 3 & Beneficiários, comunidade & Quem vai ser atingido/abrangido? & Who's going to be hit/covered? \\
\hline 4 & Objetivos & Para que se quer fazer? & What for do we want to do? \\
\hline 5 & Metas & Quanto se quer fazer? & How much do we want to do? \\
\hline 6 & Localização & Aonde se vai fazer? & Where will it be done? \\
\hline 7 & Metodologia, ações, atividades & Como se vai fazer? & How will it be done? \\
\hline 8 & Cronograma & Quando se vai fazer? & When will it be done? \\
\hline 9 & Recursos Humanos & Quem vai executar? & Who will perform? \\
\hline 10 & $\begin{array}{l}\text { Comunidade, lideranças, direção, } \\
\text { órgãos }\end{array}$ & Quem vai acompanhar? & Who will follow? \\
\hline 11 & Recursos materiais e financeiros & Com o que se vai fazer? & $\begin{array}{l}\text { With what resources are we going } \\
\text { to do? }\end{array}$ \\
\hline 12 & Fatores/condições de sucesso & $\begin{array}{l}\text { Quais são as condições } \\
\text { necessárias para que a iniciativa } \\
\text { tenha sucesso }\end{array}$ & $\begin{array}{l}\text { What are the conditions necessary } \\
\text { for the initiative to succeed? }\end{array}$ \\
\hline
\end{tabular}

Fonte: Adaptado de Meira (2003). 
Para alargamento do domínio concetual sobre campo de intervenção, têm recorrido também à utilização do Método do Quadro Lógico (MQL) que implica a aplicação da árvore de problemas e da árvore de objetivos, e em especial da Matriz de Enquadramento Lógico (MEL), de que se apresenta seguidamente um dos diversos formatos que pode assumir.

Tabela 3 Matriz de enquadramento lógico reformulada

\begin{tabular}{|c|c|c|c|c|}
\hline \multicolumn{2}{|c|}{ Matriz de Enquadramento Lógico (MEL) } & \multicolumn{2}{|c|}{$\begin{array}{l}\text { Título do projeto: } \\
\text { Duração do projeto: } \\
\text { Instituição responsável: } \\
\text { Instituições parceiras: }\end{array}$} & \multirow{2}{*}{\begin{tabular}{|l} 
Data: \\
Suposições
\end{tabular}} \\
\hline & $\begin{array}{l}\text { Lógica de } \\
\text { intervenção }\end{array}$ & Indicadores & Fontes de verificação & \\
\hline \multicolumn{5}{|c|}{$\begin{array}{l}\text { Objetivo a longo } \\
\text { prazo }\end{array}$} \\
\hline \multicolumn{5}{|l|}{$\begin{array}{l}\text { Objetivos } \\
\text { específicos }\end{array}$} \\
\hline \multicolumn{5}{|l|}{$\begin{array}{l}\text { Resultados } \\
\text { esperados }\end{array}$} \\
\hline Atividades & & $\begin{array}{l}\text { Meios } \\
\text { necessários }\end{array}$ & Custos & \\
\hline & & & & $\begin{array}{l}\text { Condições } \\
\text { prévias }\end{array}$ \\
\hline
\end{tabular}

Fonte: Adaptado de Cassiolato \& Gueresi (2010), Pfeiffer (2000), Schiefer, Monteiro \& Teixeira (2006).

Ambas as ferramentas têm sido de utilidade assinalável para concertação de posições entre intervenientes num processo de intervenção (em especial entre os membros de equipas técnicas), podendo e devendo ser utilizadas conjuntamente com outras ferramentas, o que proporciona vantagens acrescidas ao longo do processo. $^{3}$

No próximo ponto os autores descrevem duas iniciativas que consideram emblemáticas, e nas quais participaram em funções de membros de equipa técnica ou coordenação. 


\section{Práticas bem sucedidas e experiências obtidas}

Essas duas práticas são o projeto Winnet 8 (Promoção da Inovação em Igualdade de Género na Europa), e o projeto Qual_IS (Qualificar a Intervenção Social).

\section{Winnet 8}

Este foi um projeto implementado ao abrigo do Programa Inter-regional de Cooperação INTERREG IVC, que decorreu entre janeiro 2010 e dezembro 2011, e abrangeu oito países: Bulgária, Finlândia, Grécia, Itália, Polónia, Portugal, Reino Unido (Escócia), e Suécia (país promotor, através do município de Älvdalen).

Nestes países foram identificadas em regiões selecionadas as seguintes problemáticas de partida: baixa taxa de emprego feminino; discriminação laboral a nível horizontal; baixa taxa de empreendedorismo feminino; e baixas taxas de participação feminina no sector da Inovação e Tecnologia, tendo sido proposto a implementação de Women Resource Centres (WRC)/Centros de Recursos de Apoio a Mulheres (descritos por Grosso, Santos \& Baltazar, 2012), para combater essas debilidades.

É de salientar a metodologia participativa como âncora do projeto o que foi conseguido através da constituição de um Multi-Actor Group (MAG), cuja composição no caso português englobou para além de representantes das duas entidades parceiras (Universidade de Évora e Comissão de Coordenação e Desenvolvimento Regional/CCDR Alentejo), representantes de seis entidades com experiência na promoção do empreendedorismo, que aceitaram o convite que lhe foi endereçado.

\section{Qual_IS}

Projeto implementado ao abrigo do Programa Operacional Potencial Humano (POPH), tipologia 3.1.2 - Programa de Formação-Ação para entidades da Economia Social e abrangeu três Regiões de Portugal: Norte, Centro e Alentejo (sendo aqui relatada a experiência relativa ao Alentejo).

Nesta região, o objetivo do projeto consistiu em capacitar e apoiar 28 organizações sociais para o desenvolvimento de processos de qualificação organizacional, visando melhorar a eficácia da intervenção, e a promoção de respostas sociais mais adequadas para o combate à pobreza e exclusão social, e decorreu em duas fases.

Da experiência resultante da participação nestes dois projetos, há a salientar no que se refere ao projeto Winnet 8 as insuficiências na elaboração do diagnóstico, que foram em muito colmatadas com a colaboração dos representantes do MAG, que disponibilizaram informação necessária sobre boas práticas, e sinalização de 
iniciativas em curso ou em preparação que puderam assim ser potenciadas. No que concerne ao projeto Qual_IS uma das principais aprendizagens envolveu a resistência à mudança na generalidade das vinte e oito organizações em que foi implementado o plano de melhorias, pois verificou-se uma grande dificuldade em convencer alguns dirigentes e colaboradores em aderir às alterações que lhe foram propostas pelos consultores.

Após a apresentação destes dois casos que os autores têm transmitido em processos de ensino-aprendizagem, e dos quais retiram ensinamentos para adaptação em outros projetos, serão expostos no ponto seguinte as aprendizagens decorrentes da participação em vários processos de intervenção.

\section{Contributos para debate com docentes investigadores e técnicos de intervenção social}

A interação entre docentes investigadores e técnicos de intervenção incide quer sobre a fase de candidatura quer sobre a fase de concretização. Durante a concretização, com alguma periodicidade ocorrem momentos de monitorização e de avaliação.

\section{Fase da elaboração de candidaturas}

A experiência demonstrou que a leitura atenta do regulamento, nomeadamente requisitos de admissibilidade e critérios de seleção, não representa uma perda de tempo, antes constitui um investimento que só traz benefícios. A seleção de entidades parceiras adequadas é um outro pressuposto a observar, pois a sua qualificação, robustez financeira e histórico credível são fundamentais. Também questões que sendo embora mais de pormenor, mas não despiciendas, podem ser evocadas, pois representam uma regularidade que os redatores e responsáveis por candidaturas se vêm confrontados, tais como: redundância no preenchimento de vários campos do formulário (que confunde os menos experientes); diferenças de tópicos incluídos em diferentes formulários de candidatura e terminologia por vezes não esclarecida causam com frequência desorientação e desânimo, a que se pode juntar o atraso na aprovação por parte da entidade financiadora, problema que acarreta inconvenientes financeiros e pode comprometer objetivos estabelecidos e ações programadas.

Nesta fase, o papel dos interventores académicos consiste por um lado numa abordagem preventiva, alertando atempadamente os membros da equipa técnica para evitar que sejam cometidos erros evitáveis, e numa abordagem reparadora, corrigindo posteriormente os erros detetados nos itens mais sensíveis do 
formulário, e consiste ainda por outro lado, em aprofundar e disseminar o conhecimento sobre essas situações, por exemplo através de estudo de casos. Também podem e devem oportunamente promover ou participar em ações de formação (iniciativa que os autores já têm realizado) essencialmente para pessoal de organizações sem fins lucrativos.

Fase de concretização, monitorização e avaliação

Na fase de concretização surgem amiúde falhas na comunicação e articulação entre entidades parceiras, atrasos de reembolsos por parte de entidades financiadoras, e dificuldade na mobilização e motivação de beneficiários. A experiência mostra que estas insuficiências exigem que quem coordena um projeto tenha a preparação adequada para tomar medidas adequadas em tempo útil. ${ }^{4}$

É ainda fundamental que a equipa do projeto conte com os contributos dos momentos de avaliação (enquanto instrumento pedagógico), e com os contributos de momentos de monitorização (que poderá ser por exemplo trimestral) de forma a corrigir atempadamente não conformidades, e se possível melhorar pontos fortes.

Estas reflexões em torno da intervenção, conduzem a levantar a questão sobre o que se pode considerar uma intervenção bem sucedida. A experiência também mostra que uma das respostas pode apontar para que seja aquela que preenche os critérios utilizados nos diferentes momentos de avaliação (ex-ante, de acompanhamento ou ongoing, final, e de impactos), ou seja que respondendo a um problema bem formulado, se enquadre satisfatoriamente em critérios como: relevância, eficácia, eficiência, sustentabilidade e impactos (Austrian Development Agency, 2008).

Nesta fase o papel dos interventores académicos consiste no apoio à resolução de dificuldades surgidas (atividade de consultoria), e especialmente na sensibilização para os resultados dos momentos de monitorização e avaliação (que devem ser amplamente participados), cujas conclusões e recomendações devem constituir fontes de aprendizagem e de melhorias da intervenção.

Tendo este excerto sido preparado para submeter fundamentalmente à apreciação de colegas que tenham participado em processos de intervenção, com a finalidade de fomentar a reflexão a partir de experiências vivenciadas, os autores não quiseram deixar de se dirigir também mais especificamente a quem se encontra na fase de formação, deixando algumas pistas para informação, questionamento e debate. 


\section{Contributos direcionados para os recém diplomados ou finalistas dos diferentes graus de ensino em Sociologia}

Ao longo de vinte anos os autores têm tido a oportunidade de acompanhar e trabalhar em processos de intervenção com ex-alunos(as) em cuja formação de base colaboraram, tendo-se apercebido de mais valias e lacunas na sua preparação.

Confrontados com o que têm transmitido a esse público, e com a sua experiência sobre as competências que a atividade profissional requer para que graduados em Sociologia possam corresponder às expectativas e requisitos requeridos num contexto profissional altamente exigente, consideram os autores como sendo crucial evidenciar quais são as recomendações que em seu entender devem deixar para debate sobre esta questão.

Assim, num momento em que as oportunidades de emprego em funções compatíveis com o grau académico têm vindo a evidenciar dificuldades acrescidas, e as exigências para ocupação desses cargos são elevadas, é de considerar as designadas competências técnicas (hard skills), mas também as competências não técnicas ou comportamentais (soft skills).

De entre as competências técnicas há a destacar as que se centram no domínio dos Métodos e Técnicas de Investigação, as que se prendem com o Planeamento, Monitorização e Avaliação de projetos (de incontornável importância para o exercício de atividade profissional em intervenção social, porque nos momentos em que são requeridas facultam informação e orientações imprescindíveis para o sucesso de um projeto). Igualmente importante são as técnicas que se centram no âmbito das Tecnologias de Informação e Comunicação (na ótica do utilizador). Sublinha-se a importância destas competências serem exercitadas e reforçadas para que além do que é ministrado nas atividades curriculares, devendo os docentes e discentes preocuparem-se em reforçar os conhecimentos, através por exemplo de atividades extra curriculares que concorram para uma melhor preparação dos futuros candidatos a um cargo profissional na intervenção social.

Referindo as competências não técnicas, tem-se vindo a acentuar o seu significado, sendo atualmente concedida especial atenção a esta dimensão dos recursos humanos.

Um dos conjuntos que foi considerado significativo inclui as seguintes competências: resolução de problemas complexos; pensamento crítico; criatividade; inteligência emocional; capacidade de julgamento e de tomada de decisões; flexibilidade cognitiva; negociação; gestão de pessoas; espírito colaborativo; e orientação para o utente (World Economic Forum, 2016).

Num outro conjunto, são referidas as sete atitudes que se seguem: atitude positiva; resiliência; resolução de problemas; gestão do tempo; capacidade de comunicação; trabalho em equipa; e potencial de aprendizagem (Santiago, 2014). 
Esta é uma informação que se impõe pelo menos divulgar junto dos futuros profissionais, sensibilizando-os para uma matéria que é do seu interesse.

\section{Conclusões}

A sistematização de apontamentos e reflexões proporcionados pelo desempenho de funções em diferentes projetos, assim como a análise da bibliografia consultada, permitiram relevar práticas bem-sucedidas e insucessos, lições aprendidas mais significativas e relacionar a relevância de conhecimentos proporcionados durante a formação de base, e mesmo da formação pós graduada, bem como apontar insuficiências que podem ser colmatadas durante a aprendizagem curricular, aprendizagem extra curricular, e pós escolar.

Procurou-se assim contribuir com informação para que os diplomados em Sociologia correspondam às exigências com que se defrontam ao participar em projetos de intervenção social, sendo também referidas dificuldades de inserção dos recém-diplomados no mercado de trabalho, em especial na área social, e apontadas algumas sugestões para minimizar essas dificuldades.

Podendo fazer-se a distinção entre sociologia pura (ou básica) que tem o objetivo de descobrir "como funciona a sociedade" (que tem de ser devidamente considerada), e a sociologia aplicada que procura entender os conhecimentos adquiridos na sociologia básica e utilizá-los na resolução de problemas de natureza prática ou social, a experiência mostra que os conhecimentos adquiridos na formação de base nomeadamente em métodos e técnicas de investigação, reforçados com conhecimentos de informática na óptica do utilizador e com o conhecimento em disciplinas complementares: demografia, economia, planeamento, são fulcrais.

Porém tem-se acentuado nestes últimos anos a importância das competências não técnicas, questão para a qual os futuros profissionais e aqueles que participam na sua formação devem ter na devida consideração, para que seja conseguido e reconhecido o papel de sociólogos em projetos de intervenção social.

Esta preocupação e a atividade de apoio técnico-científico prestada em diversas intervenções tem conduzido a que sejam revistos e adaptados pelos autores, a orientação geral e os tópicos que integram os curricula, de forma a corresponderem às exigências que a dinâmica da intervenção coloca (no que se refere por exemplo à participação de beneficiários e intervenientes, e à utilização de procedimentos técnicos já testados). Estas preocupações levam a, chamar pontualmente colaboradores no processo de ensino aprendizagem cujo percurso considerável lhes possibilita transmitir e debater lições aprendidas, e assim contribuir para a qualificação e empregabilidade dos futuros profissionais diplomados em Sociologia. 


\section{Notas}

1 Dos quais os seguintes não são descritos: i) Planificação de Projetos Orientados por Objetivos (PPOO) e, ii) Plano de Implementação Orientada por Objetivos (PIOO). Para além destes foram ainda recenseados os seguintes: Gestão Baseada em Resultados (Results-Based Management RBM), Gestão Participativa de Projetos (GESPAR), Intervenção Participativa de Atores (INPA), Método CENDES-OPS (Centro de Estudos de Desenvolvimento/Organização PanAmericana de Saúde), Participative Monitoring Impact (PMI), Participatory Learning and Action (PLA), Planejamento Estratégico Democrático (PED), Programação de Gestão por Resultados da Atenção Básica (Prograb).

2 Esta é uma das ferramenta da qualidade que pode ser utilizada em qualquer intervenção, com a finalidade de anotar de maneira organizada e planeada como serão concretizadas as ações gizadas, assim como por quem, quando, onde, por quê, como, e também qual será o custo dessa intervenção, sendo o significado das siglas o seguinte: What (o quê); Who (quem); When (quando); Where (onde); Why (por que); How (como); How Much (quanto).

3 Como por exemplo: Balanced scorecard, Mapas estratégicos, Análise estratégica (Análise de stakeholders, Análise contextual, Análise organizacional e Análise SWOT), Carta de ofertas e procuras (parcerias e redes), e Plano de atividades.

4 O que requer um perfil que inclua aptidões técnicas, interpessoais e conceptuais $(\mathrm{Cu}-$ nha et al., 2014).

Por decisão pessoal, os autores do texto escrevem segundo o novo acordo ortográfico.

\section{Referências}

Almeida, J. (2001). Em defesa da investigação-acção. Sociologia, Problemas e Práticas, (37), 175-176.

Austrian Development Agency. (2008). Avaliações de projectos e programas. Viena:

Departamento de avaliação da ADA. Retrieved from

http:/ / www.oecd.org/development/evaluation/dcdndep/47069570.pdf

Bourdieu, P. (2001). Para uma sociologia da ciência. Lisboa: Edições 70.

Cassiolato, M., \& Gueresi, S. (2010). Como elaborar modelo lógico: Roteiro para formular programas e organizar avaliação. Brasília: Instituto Econômico de Pesquisa Aplicada. Retrieved from http://www.ipardes.gov.br/pdf/multissetorial/nota_tecnica_IPEA.pdf

Cunha, M. N., Rego, A., Cunha, R. C., Cardoso, C. C., \& Neves, P. (2014). Manual de comportamento organizacional e gestão. Lisboa: RH Editora.

Grosso, L., Santos, M., \& Baltazar, M. (2012). Modelos de centros de recursos para mulheres na Europa. In S. Baltazar, M. Santos, \& F. Sabino (Orgs.). Empreendedorismo, igualdade de género e desenvolvimento regional e local (pp.139-146). Casal de Cambra: Colibri. 
Guerra, I. (2003). Polémicas e modelos para uma sociologia de intervenção. Cadernos de Estudos Africanos, (4), 71-83.

Herreros, G. (2013). L'intervention en sociologie: Cadres et pratiques. Communication dans Journée d'étude de l'Association des professionnels en Sociologie de l'entreprise (APSE), France.

Mannhein, K. (1974). O problema de uma sociologia do conhecimento. In R. Bertelli, et al. (Orgs.), Sociologia do conhecimento (pp. 100-115). Rio de Janeiro: Zahar.

Meira, R. C. (2003). As ferramentas para a melhoria da qualidade. Porto Alegre: SEBRAE.

Moreau, D. (2015). Intervention sociology: History and foundations. International Review of Sociology, 25(1), 180-193.

Pfeiffer, P. (2000). O quadro lógico: Um método para planejar e gerenciar mudanças. Revista do Serviço Público, 51(1), 81-122. Retrieved from http:/ / www.nescon.medicina.ufmg.br/biblioteca/imagem/0196.pdf

Santiago, A. (2014, 21 de fevereiro). Sete soft skills para uma carreira de sucesso. Expresso (online). Retrieved from

http:/ /expresso.sapo.pt/blogues/bloguet_economia/blogue_vipp_valorizacao_i mg_pessoal_profissional/sete-soft-skills-para-uma-carreira-de-sucesso $=\mathbf{f} 857340$

Santos, M. (2011). Planeamento: Noções introdutórias. Retrieved from http://home.uevora.pt / mosantos/download/Planeamento_NocoesIntrodutorias_28Jul11Schiefer, U., Monteiro, S., \& Teixeira, P. J. (2006). MAPA - Manual de facilitação para a gestão de eventos e processos. Cascais: Principia.

Trilla, J. (1998). La educación fuera de la escuela. Barcelona: Ariel.

Vranken, D., \& Olgierd, K. (Eds.) (2001). La sociologie et l'intervention, ouvertures sociologiques. Louvain-la-Neuve: De Boeck Université.

World Economic Forum. (2016). The future of jobs (Report). Geneva: WEC.

Marcos Santos. Doutorado em Sociologia Rural e Urbana. Investigador integrado, Centro Interdisciplinar de Ciências Sociais (CICS.NOVA.UÉvora).

Maria da Saudade Baltazar. Doutorada em Sociologia. Departamento de Sociologia, Escola de Ciências Sociais. Investigadora integrada, Centro Interdisciplinar de Ciências Sociais (CICS.NOVA.UÉvora).

Data de submissão: 25/07/2017 | Data de aceitação: 14/10/2017 